\title{
Une perspective philosophique sur la durabilité forte. Pour un écocentrisme relationnel
}

A philosophical approach of strong sustainability. In defense of relational ecocentrism

\section{Rémi Beau}

\section{OpenEdition}

\section{Journals}

Édition électronique

URL : http://journals.openedition.org/developpementdurable/13613

DOI : 10.4000/developpementdurable.13613

ISSN : 1772-9971

Éditeur

Association DD\&T

Référence électronique

Rémi Beau, «Une perspective philosophique sur la durabilité forte. Pour un écocentrisme relationnel», Développement durable et territoires [En ligne], Vol. 10, $n^{\circ} 1$ | Avril 2019, mis en ligne le 04 avril 2019, consulté le 20 avril 2019. URL : http://journals.openedition.org/developpementdurable/13613 ; DOI : 10.4000/developpementdurable.13613

Ce document a été généré automatiquement le 20 avril 2019.

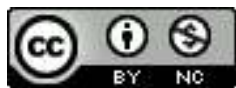

Développement Durable et Territoires est mis à disposition selon les termes de la licence Creative Commons Attribution - Pas d'Utilisation Commerciale 4.0 International. 


\title{
Une perspective philosophique sur la durabilité forte. Pour un écocentrisme relationnel
}

\author{
A philosophical approach of strong sustainability. In defense of relational \\ ecocentrism
}

Rémi Beau

1 Les éthiques environnementales de langue anglaise ont initialement réservé un accueil pour le moins mitigé au débat sur les durabilités "forte» et "faible», tel qu'il fut notamment présenté par Herman Daly et John Cobb (1989). Pour bon nombre des philosophes de ce courant, qui étaient engagés depuis les années 1970 dans des discussions théoriques sur la valeur intrinsèque de la nature (Larrère, 1997 ; Hess, 2013), ce débat était une querelle d'économistes et la réflexion sur la "durabilité forte » se montrait d'une radicalité insuffisante pour rejoindre leurs propres intérêts de recherches qui visaient à établir des obligations morales à l'égard de la nature indépendantes des intérêts humains (Jamieson, 1998). En somme, il apparaissait que les éthiques du respect de la nature se situaient bien au-delà de la réflexion sur la durabilité sur une échelle caractérisant l'engagement en faveur de la préservation des espaces et des espèces naturels. Mais, si la théorisation de la valeur intrinsèque de la nature conduisait logiquement les éthiciens à s'intéresser principalement à des espaces sauvages dont les hommes étaient absents, suivant l'idée classique de «wilderness » (Nash, 1967), le paysage des éthiques environnementales s'est progressivement transformé dans les années 1990 et a accordé une place croissante à la réflexion sur les espaces habités par les hommes. Critiquant le dualisme qui sous-tendait la valorisation exclusive de la nature sauvage séparée des hommes, des éthiques environnementales relationnelles ont affirmé leur intérêt pour des formes de nature plus ordinaires (Beau, 2017) et orienté leurs recherches en direction des manières de cohabiter avec des acteurs non humains sur un espace commun. 
2 Après avoir décrit l'écart qui semblait exister entre les approches économiques de la durabilité et les éthiques environnementales, nous chercherons à montrer comment le développement de ces approches relationnelles conduit certaines de ces éthiques à revenir sur le terrain de la réflexion sur la durabilité. Parmi ces dernières, nous nous intéresserons en particulier à l'éthique écocentrique du philosophe américain Baird Callicott ainsi qu'à l'éthique relationnelle de la philosophe australienne Val Plumwood, afin de montrer comment celles-ci permettent de défendre une approche philosophique de la durabilité forte et écologique. En discutant à cette occasion une critique importante visant sous un angle politique la valorisation des relations en matière d'éthique environnementale, nous dessinerons les contours d'un écocentrisme relationnel qui prend au sérieux la question de l'insubstituabilité de la nature.

\section{La réception du débat sur les durabilités faibles et fortes au sein des éthiques environnementales}

\subsection{Le débat entre durabilités faibles et fortes}

3 La distinction entre des versions fortes et faibles de la durabilité naît à la toute fin des années 1980 de la critique menée par des économistes (Cobb et Daly, 1989; Costanza, 1991; Martinez-Alier, 1990) qui ne se satisfaisaient pas de la réponse apportée par certains de leurs collègues néo-classiques à la remise en cause du modèle croissanciste. Aux auteurs du rapport Meadows (Meadows et al., 1972) qui exposaient l'idée que la poursuite d'une croissance exponentielle dans un monde fini conduisait à la catastrophe, ces derniers avaient, en effet, répliqué que la crise écologique exigeait sans doute un réajustement du modèle économique capitaliste, mais qu'il n'en ébranlait aucunement les fondements néo-classiques (Solow, 1974). C'est de façon générale la direction suivie par une économie de l'environnement ${ }^{1}$ se donnant comme objectif principal l'internalisation des externalités négatives du développement des sociétés industrielles (Barde, 1992). Fondant leur analyse sur la distinction entre trois types de capitaux soutenant la capacité productive (le capital physique, le capital humain et le capital naturel), ces économistes défendent l'idée que l'impératif de durabilité porte sur la somme totale de ces différents stocks de capitaux. De ce point de vue, le risque d'épuisement de certaines ressources naturelles n'est pas en lui-même problématique. Il le devient seulement s'il entraîne dans le temps la diminution de la somme totale des capitaux dont dispose une génération pour répondre à ses besoins, c'est-à-dire si l'érosion du capital naturel n'est pas compensée par une élévation des deux autres types de capitaux. Le contenu normatif de cette « durabilité faible » se réduit donc à l'obligation adressée à la génération présente de trouver des substituts aux ressources naturelles qu'elle tend à épuiser. Cette voie repose ainsi sur une hypothèse forte, celle de la substituabilité totale du capital naturel et du capital humainement produit : il n'y aurait en définitive rien d'insubstituable en droit dans la nature (Solow, 1993). Or, de ce positionnement à la négation pure et simple du caractère problématique de l'épuisement des ressources naturelles, il n'y a qu'un pas, qu'un auteur comme Wilfred Beckerman n'hésite pas à franchir (Beckerman, 1994, 1995).

Cette réponse n'est évidemment pas satisfaisante pour les critiques de la durabilité faible qui contestent cet axiome de la substituabilité totale du capital naturel et lui opposent une conception de la durabilité forte (Cobb et Daly, 1989). Pour Cobb et Daly, ce qui est absurde, c'est de croire que l'on peut toujours substituer du capital humainement 
construit à des ressources naturelles. Cela consiste à confondre systématiquement les gains en efficacité que permettent certains développements techniques avec la substitution d'un capital technique à une ressource naturelle (Daly, 1995). Seul le formalisme abstrait de l'économie néo-classique peut occulter la nécessaire complémentarité du capital naturel et du capital humainement produit. Par conséquent, les tenants de la durabilité forte soutiennent que l'impératif de durabilité ne doit pas porter simplement sur la somme des stocks de capitaux, mais bien sur un stock de capital naturel lui-même, qu'il faut maintenir au-dessus d'un seuil critique (Ekins, 2003). De ce point de vue, tandis que la version faible de la durabilité reste indéterminée quant à la nature des choses elles-mêmes qu'il s'agissait de faire perdurer, la version forte exige la conservation de ressources et de services naturels, car il y a bien de l'insubstituable dans la nature qui doit être transmis aux générations futures.

\subsection{Le regard porté par les éthiques environnementales sur ce " débat d'économistes "}

5 Nées au début des années 1970, les éthiques environnementales ont une quinzaine d'années d'existence lorsque la notion de «développement durable » apparaît véritablement dans l'espace public (Brundtland, 1987). La jeune discipline philosophique s'est entre-temps structurée en différents sous-courants de pensée qui incarnent autant de propositions de réponses à la question de savoir comment dépasser l'anthropocentrisme étroit qui caractérise les théories morales de l'Occident moderne. De l'anthropocentrisme élargi de Bryan Norton (Norton, 1984) à l'écocentrisme de Baird Callicott (Callicott, 1989, 1999), en passant par le biocentrisme de Paul Taylor (Taylor, 1986), l'éthique naturaliste de Holmes Rolston (Rolston, 1989) ou encore l'écosophie d'Arne Naess (Naess, 1989), les éthiques environnementales ont développé une large palette de réflexions sur les valeurs de la nature ${ }^{2}$, s'efforçant de caractériser la nature de ces valeurs, de définir ceux qui en sont les porteurs, mais aussi de décrire les obligations morales directes qui leur sont associées. Fructueuses sur le plan théorique, ces recherches firent néanmoins face, à partir de la fin des années 1980, à une critique importante pointant du doigt l'incapacité qui aurait été celle de l'éthique environnementale à peser véritablement sur les politiques écologiques et à se traduire pratiquement par des mesures en faveur de la protection de l'environnement (Katz et Light, 1996). Sous cet angle la radicalité théorique de ce décentrement de la morale était jugée à la fois trop clivante et trop éloignée des oppositions politiques réelles pour peser dans l'espace public des sociétés démocratiques (Norton, 2008). C'est précisément de ce point de vue que le développement durable, défini comme un cadre opérant pour les politiques publiques, ne pouvait que retenir l'attention de ces penseurs à la recherche de modalités de mise en pratique de leurs réflexions théoriques.

6 Et c'est bien dans ce sens que, dans les années 1990, plusieurs philosophes de l'environnement vont se pencher sur la notion de développement durable et sur le débat sur la durabilité, afin d'examiner si celui-ci est en mesure d'apporter un éclairage sur la traduction dans des politiques publiques de l'appel qu'ils avaient lancé en faveur du dépassement de l'anthropocentrisme moral. Sous le premier aspect du rassemblement le plus large possible pour faire avancer la cause écologiste, le développement durable semblait tout indiqué. CEuvrer au décloisonnement du traitement des questions écologiques et sociales, dépasser l'opposition largement factice entre le développement 
des Suds et la protection de l'environnement constituaient des "promesses» qui appelaient au rassemblement le plus large. Mais, ce niveau élevé de généralité ne manquait pas également d'éveiller un fort soupçon, celui que l'expression soit en réalité le nom d'un consensus creux sur le plan normatif (Noss, 1991 ; Jamieson, 1998) ou encore celui d'un «grand parapluie vert » qui obscurcissait plutôt qu'il n'éclairait les enjeux environnementaux (Rolston III, 2002a), voire tendait même à relativiser l'urgence écologique (Plumwood, 2002). Appliquant leur propre grille d'analyse à ce débat, les éthiciens de l'environnement cherchèrent ainsi à identifier parmi les réponses apportées des propositions susceptibles de contribuer à la discussion qui les occupait principalement, celle de la définition d'obligations directes à l'égard de la nature, c'est-àdire des obligations qui ne se rapportent pas en dernière instance à la satisfaction d'intérêts humains.

7 En ce qui concerne les tenants de la durabilité faible, l'affaire est rapidement entendue. La position défendue par ces auteurs est bien plus proche de celles combattues par les éthiques environnementales que de celles qu'elles soutiennent. Leur adhésion pleine et entière au cadre néo-classique les classe, en effet, parmi les pensées relevant de cet anthropocentrisme étroit qui consiste à ne mesurer le monde qu'à l'aune de l'humain (Larrère, 2002). La thèse de la substituabilité totale de la nature incarne de façon emblématique une pensée du rapport démiurgique des hommes à la nature. Elle est l'expression de la croyance forte que l'ingéniosité technique des hommes les dote d'une capacité de fabrication illimitée des ressources qui leur sont nécessaires (Callicott, 2016 : 314).

8 C'est donc logiquement vers les défenseurs de la durabilité forte que se tournent les éthiciens de l'environnement. Parmi ces derniers, le philosophe Bryan Norton (2005) se livre à un examen détaillé de la position défendue par Daly et Cobb (1989). S'appuyant sur une discussion entretenue avec le spécialiste de philosophie morale et politique Brian Barry, Norton propose de différencier les approches de la durabilité selon qu'elles visent le bien-être humain ou la préservation de « choses » [stuff] naturelles (Norton, 2005 : 306). Or, pour le philosophe, toutes les approches purement économiques de la durabilité se classent en dernière instance parmi les premières (Norton, 2003, 2005). Elles n'échappent pas à la « grande simplification » (Norton, 2003) qui réduit la diversité des relations entre les hommes et la nature à des rapports de valorisation économique monétairement mesurables. La question pour Norton est de comprendre la façon dont une communauté peut s'engager dans une trajectoire de durabilité forte en instituant l'insubstituabilité de certaines «choses" naturelles [natural stuff]. Et cela ne peut passer, selon lui, que par la construction d'un accord politique sur les éléments de la nature qu'une communauté désire «projeter dans le futur » (Norton, $2003:$ 446). De ce point de vue, la distance qui sépare les approches économiques des éthiques environnementales tient au fait que les premières débattent d'une insubstituabilité de fait quand les secondes défendent une insubstituabilité de droit. Il s'agit, d'un côté, d'une interrogation sur les possibilités réelles de trouver des substituts à des ressources ou à des services naturels et, de l'autre, sur ce que nous devons rendre durable, parce que nous lui attribuons une valeur qui ne se réduit pas à la valeur économique (Jamieson, 1998).

9 Le regard que porte Norton sur les approches économiques de la durabilité forte est d'autant plus intéressant qu'il offre un éclairage plus large sur la réception du débat au sein des éthiques environnementales. En effet, dans la mesure où Norton est le représentant dans ce dernier courant d'une aile modérée en ce qui concerne le rejet de 
l'anthropocentrisme (Norton, 2008), celle du pragmatisme environnemental, nous pouvons considérer que les critiques qu'il adresse à l'approche économique de la durabilité visant l'insuffisance de la place qu'elle accorde aux intérêts de la nature sont partagées par ses collègues plus radicalement non anthropocentristes ${ }^{3}$. Cette critique semble bien exprimer le positionnement général des éthiciens de l'environnement devant des analyses qui partent du postulat initial que la notion de «capital naturel » est suffisamment inclusive pour intégrer les objectifs de la durabilitét. L'expression ellemême ne peut, en effet, à première vue que laisser sceptiques ces auteurs travaillant à la déconstruction de l'anthropocentrisme étroit qui caractérise selon eux les raisonnements économiques (Jamieson, 1998 ; Holland, 2002 ; Vucetich et Nelson, 2010). Penser la part insubstituable de la nature ${ }^{5}$ sous l'appellation de capital naturel, c'est la référer à des usages humains sous la modalité particulière d'un rapport de valorisation économique. Ne risque-t-on pas dans ce cadre de laisser à l'écart une partie de ce qu'il s'agirait de voir perdurer selon une approche véritablement écologique de la durabilité ? C'est bien ce que suggérait déjà la figure tutélaire de l'éthique environnementale, le forestier américain Aldo Leopold, qui écrivait dans une page célèbre de l'Almanach d'un comté des sables :

«La montagne qu'il faut déplacer pour libérer le processus vers une éthique, c'est tout simplement ceci : cessez de penser au bon usage de la terre comme à un problème exclusivement économique » (Leopold, $2000: 283$ ).

\subsection{Un accord objectif surprenant?}

Ainsi, l'examen rapide des réflexions sur la durabilité, proposées par quelques-uns des principaux auteurs de l'éthique environnementale, permet de constater qu'ils paraissent s'accorder à penser que ce débat d'économistes reste assez éloigné de leurs propres objets et objectifs de recherche. Autrement dit, parce qu'ils aspirent précisément à soustraire la préservation de la nature du champ d'application de la rationalité économique, ces philosophes seraient par avance peu disposés à dialoguer avec les économistes, y compris avec ceux d'entre eux qui défendent une version de la durabilité moins faible que leurs collègues néo-classiques. Mais, de façon étonnante, ce positionnement par rapport aux tenants de la durabilité forte semble conduire à la conclusion qu'un accord objectif pourrait réunir, en définitive, les éthiciens de l'environnement et un défenseur de la durabilité faible comme Robert Solow. Ces auteurs s'accorderaient, en effet, à penser que la question de la préservation de la nature ne doit pas être abordée sous l'angle de la durabilité (Solow, 1993). Il y a là une manière de renvoyer chacun à la tâche qui serait la sienne: aux économistes, la réflexion sur les façons de rendre le développement des sociétés humaines " durable "; aux éthiciens, le travail conceptuel visant à mettre au jour des raisons non économiques de préserver des pans de nature sauvage. Mais, n'est-ce pas là aussi une manière assez commode de rendre parfaitement inoffensive la critique des modes de développement des sociétés contemporaines portée par ces philosophes depuis les années 1970 ? L'éthique environnementale peut-elle ainsi réellement se contenter de penser la valeur des espaces épargnés par le déploiement des activités industrielles? 


\section{Des éthiques de la valeur intrinsèque aux éthiques de la nature relationnelles}

\subsection{Un « tournant relationnel » vers la nature proche}

On a souvent souligné à juste titre l'importance de l'idée américaine de wilderness (Nash, 1967) pour le courant des éthiques environnementales. Parce qu'elles conduisaient une réflexion sur la possibilité de reconnaître une valeur morale indépendante des seuls intérêts humains, ces éthiques ont en effet initialement porté leur regard sur les espaces et les êtres se situant à l'écart des activités humaines, sur une nature dont les hommes sont pour l'essentiel absents. Qu'elles portent sur la nature qui aurait précédé les sociétés humaines, dont la wilderness constituerait les derniers fragments, ou sur la nature qui survivra à l'humanité, comme c'est le cas dans les expériences de pensée sur le dernier homme, les discussions menées poursuivent une même interrogation sur la présence de valeurs dans un monde en deçà ou au-delà de l'humain (Light et Rolston, 2002). Comme le décrit l'un de ses principaux initiateurs, le philosophe américain Holmes Roslton, il s'agissait de faire prendre à la philosophie un premier tournant vers la nature sauvage (Rolston, 1989).

Mais, si cette focalisation des éthiques environnementales sur la nature sauvage, pensée comme une nature extérieure, éloignée, a indéniablement marqué les deux premières décennies de leur développement, celle-ci a par la suite fait l'objet de discussions importantes au sein de la discipline. Et ce débat a été l'occasion de revenir sur la conception classique de l'idée de wilderness, qui reçut de fortes critiques (Callicott et Nelson, 1998, 2008). Parmi ces dernières, c'est sans doute celle que développa l'historien William Cronon qui fit le plus grand bruit, avec la parution d'un article dans lequel il posait les enjeux du « problème » de la wilderness (Cronon, 1996). Il y mettait en place un argument qui sera abondamment repris par la suite, celui du caractère culturellement construit et artificiel de la wilderness.

13 Selon ces critiques, il fallait donc se départir de cette idée classique de wilderness pour penser une nouvelle conception des éthiques de la nature. Sortir de ce cadre théorique servait, en effet, l'objectif d'attirer l'attention sur des formes de nature plus ordinaires, une nature avec laquelle les hommes vivent et interagissent, et à laquelle les pensées de la wilderness ne s'intéressaient pas (Beau, 2017). Parce qu'elles en seraient restées à un cadre dualiste qui oppose les hommes et la nature, les éthiques du respect de la wilderness ne pouvaient pas s'appliquer aux relations communes entre les hommes et la nature (Plumwood, 1998 : 671).

14 L'un des objectifs qui émerge donc de ce débat sur la wilderness est de développer des approches de l'éthique environnementale qui s'efforcent d'étendre leur portée pratique aux interactions entre les hommes et la nature. Schématiquement, cela consiste à passer des éthiques du respect de la nature à ce que Carolyn Merchant décrit comme des éthiques du partenariat avec la nature, qui cherchent à définir ce que peuvent être de bonnes relations ou des formes de cohabitation plus réussies avec la nature (Merchant, 2004). Ce débat fut plus particulièrement l'occasion pour des éthiciens de l'environnement comme Val Plumwood et Baird Callicott de plaider en faveur de ce que l'on peut décrire comme des éthiques relationnelles de la nature (Beau, 2017). 


\subsection{Une approche philosophique de la durabilité}

15 Ces approches permettent-elles d'envisager à nouveaux frais le dialogue entre les éthiques environnementales et certains théoriciens de la durabilité ? En mettant en avant leur intérêt pour les pratiques quotidiennes, les manières d'habiter un espace ou plus généralement pour les modes de vie, les éthiques de la nature brouillent les frontières de la carte trop simple dessinée par Solow. Elles montrent ainsi que ce qui les sépare d'une théorie de la durabilité faible n'est pas tant leurs objets de recherches que leurs façons respectives de les appréhender.

16 C'est sans doute Baird Callicott qui se montre le plus clair sur la façon dont cette inflexion donnée à l'éthique environnementale peut reconduire sur le terrain de la durabilité. Intitulant, en effet, un article "The wilderness Idea Revisited. The sustainable development alternative », celui-ci propose, dès 1991, une interprétation écologique du développement durable, qu'il définit de la façon suivante :

«Par "développement durable", je veux dire le développement d'une activité économique humaine qui est limitée par des exigences écologiques; une activité économique qui ne compromet pas gravement l'intégrité écologique; et, idéalement, une activité économique qui améliore la santé des écosystèmes » (Callicott, $1998: 355$ ).

17 Ainsi, refusant de s'en tenir à la critique de la notion de durabilité, Callicott s'attache à définir ce qui pourrait constituer une approche écologiquement forte de la durabilité ou une «durabilité écologique» (Callicott et Mumford, 1997 ; Callicott, 2016). Loin de ses interprétations "développementistes", celle-ci permettrait de penser la durabilité comme un véritable outil pour la conservation de la nature (Callicott et Mumford, 1997) et un cadre permettant d'identifier ce que seraient des interactions mutuellement avantageuses pour les hommes et la nature (Callicott, 1998). Sans se substituer nécessairement aux politiques de préservation, les objectifs de la durabilité écologique s'étendraient aux espaces habités ou transformés par les hommes. Pour ces derniers, il ne s'agirait pas de viser la préservation de leur intégrité, c'est-à-dire celui d'un état écologique de référence, mais de maintenir la santé fonctionnelle des écosystèmes qu'ils abritent en favorisant les relations positives à cet espace (Callicott et Mumford, 1997).

Mais, si cette transformation qui mène du «développement durable » à la "durabilité écologique » procède bien d'un travail critique mené par Callicott, elle témoigne aussi d'une forme d'optimisme quant à la capacité de la notion à éclairer et à favoriser le développement de sociétés moins destructrices de leur environnement. Dans ce sens, le philosophe écrivait au début des années 1990 :

« Nous pouvons, j'en suis convaincu, construire notre propre société durable, postmoderne, technologiquement avancée et scientifiquement informée tout comme par le passé les Minoens en Méditerranée, les agriculteurs traditionnels en Europe de l'Ouest, et les Incas dans les Andes avaient construit les leurs » (Callicott, 1998 : 357).

Or, ce jugement n'était-il pas en définitive trop optimiste ? Et, au fond, ce déplacement du centre d'intérêt de l'éthique environnementale de la nature sauvage vers les espaces habités par les hommes ne s'accompagnait-il pas de la perte d'une part du mordant de sa critique écologique? C'est en tout cas ce que suggère une critique qui a émergé des développements récents de la philosophie environnementale et qui reconduit en quelque sorte vers le débat sur la durabilité forte. 


\section{L'éthique environnementale entre durabilités fortes et faibles}

\subsection{Une critique aiguisée des éthiques relationnelles de la nature}

La réaction critique face aux détracteurs de l'idée de wilderness ne s'était pas fait attendre au sein du courant environnementaliste américain. Emmenés sur le plan philosophique par Holmes Rolston, l'un des maîtres à penser de l'éthique environnementale, bon nombre d'auteurs voulurent immédiatement opposer aux défenseurs du dépassement du cadre théorique moderne les vertus théoriques et pratiques du dualisme de l'homme et de la nature dans l'optique de protéger cette dernière du développement accéléré des activités humaines (Rolston III, 2008, 2002b). Depuis les années 2010, dans un contexte politico-économique de plus en plus hostile à l'écologisme, cette « contre-critique » s'est aiguisée et renforcée ${ }^{6}$, décrivant plus explicitement les risques de fragilisation des politiques de conservation de la nature qui naissent de ce «tournant relationnel » des éthiques environnementales (Arpin, 2016).

Dans son ouvrage récent, le philosophe Frédéric Neyrat (2016) expose de façon très claire les enjeux de cette critique d'un environnementalisme qui veut penser, par-delà les dualismes, les interconnexions entre les hommes et la nature. En refusant d'appréhender la nature comme une extériorité radicale, l'écologie du "tout relié » s'engagerait, sans s'en rendre compte, sur une pente glissante qui conduirait à une forme d'anaturalisme (Neyrat, 2017), c'est-à-dire à la négation pure et simple de la nature. En définitive, la déconstruction du dualisme de l'homme et de la nature, à laquelle participent les approches relationnelles, aurait accompagné la montée en puissance des nouveaux thuriféraires de la mort de la nature, cette position qui consiste à nier précisément la part de la terre non construite et inconstructible par les hommes. Neyrat écrit dans ce sens :

«Si donc le bio- ou l'écocentrisme de l'écologie profonde échoue à remettre convenablement le statut sujet-objet dominant (l'anthropocentrisme), c'est parce qu'il est incapable de contester la répartition qui le sous-tend, cet appel à l'unité ou cette affirmation inconditionnelle de l'interconnexion de tout avec tout » (Neyrat, $2016: 254)$.

Les critiques de l'idée de wilderness qui invitaient à s'intéresser à la nature proche et ordinaire ne pourraient en définitive s'opposer à la montée en puissance de l'écoconstructivisme et auraient même en fin de compte rendu le terrain fertile à la croissance de la mouvance écomoderniste et géo-constructivistes (Shellenberger et Nordhaus, 2011 ; Asafu-Adjaye et al., 2015).

Passer de la préservation de l'intégrité d'un espace naturel au maintien de la santé fonctionnelle des écosystèmes, comme invitait à le faire Baird Callicott, ce serait affaiblir la protection de la nature. Comme le résume Frédéric Neyrat (2016:28), «si tout change, alors pourquoi s'opposer à quelque changement économique que ce soit? $\mathrm{Et}$, aux penseurs critiques de la wilderness, ce dernier adresse l'objection suivante :

« [Il] ne suffit pas de dire que la "wildness (en tant qu'opposé à la wilderness) peut être trouvée partout" car, dès lors, pourquoi se soucier de la protéger en quelque endroit du monde ? Pourquoi s'opposer à la transformation des parcs nationaux en terrains d'exploitation d'énergie extrême? » (Neyrat, 2016 : 289). 
ainsi que la question de la durabilité forte réapparait, car la part inconstructible de la terre que veut défendre Frédéric Neyrat relève aussi de cet insubstituable que les théoriciens de la durabilité faible ne voulaient pas reconnaître. De ce point de vue, si l'on mène jusqu'à son terme cette critique d'une écologie du "tout relié », qui se serait en définitive rendue elle-même incapable de reconnaître les limites de la substituabilité de la nature, elle conduit bien à souligner ses affinités avec une conception faible de la durabilité. C'est ce que ne manque pas de relever l'historien Christophe Bonneuil en soulignant comment dans ce passage d'une ontologie moderne dualiste à une ontologie postmoderne pourrait bien s'exprimer une nouvelle forme de déni des limites de la maitrise humaine (Bonneuil, 2015).

En réalité, l'idée qui se met ainsi en place ici est que les éthiciens de l'environnement qui ont mené la critique de la wilderness n'ont pas su voir que le contexte politico-économique dans lequel ils s'exprimaient annonçait une alternative dans laquelle rejeter une position consistait, nécessairement, même sans le vouloir, à endosser l'autre. Cette alternative peut s'énoncer de la façon suivante :

«L'ancien environnementalisme est alors dénigré pour avoir commis l'erreur de croire à une nature vierge, existant en dehors des humains, alors que, selon le nouvel esprit (constructiviste et connexionniste) du capitalisme (vert), la nature serait réseau, hybride (tout comme l'être humain lui-même dans la radicalisation transhumaniste de ce courant) » (Bonneuil, 2015).

Bien peu de choses sépareraient donc le refus du dualisme de l'adhésion à l'hyperconnexionnisme. Plus précisément, dans un climat anthropocénique où l'hybridisme absorbe et dissout les critiques avec une efficacité redoutable, les éthiques relationnelles qui veulent se situer entre ces deux positions semblent de moins en moins audibles. Au fond, à mesure que les remparts cèdent face à la financiarisation et à l'exploitation généralisée de la nature, les concepts qui auraient pu servir à s'opposer à ce processus sont comme emportés, et avec eux ceux qui voulaient les utiliser, du développement durable, à la compensation écologique, en passant par les services écosystémiques (Maris, 2014). Dans un tel contexte, on ne peut, en effet, que comprendre le goût des lignes claires qui délimitent les fronts écologiques encore identifiables (Maris, 2015).

\subsection{Vers une approche écocentrique de la durabilité forte}

Néanmoins, on peut également se demander si la reconduction d'un dualisme appuyant la nécessité de préserver ce qu'il reste de la nature ne conduit pas à écarter une piste, sans doute insuffisamment explorée, mais qu'indiquent pourtant certains penseurs de l'éthique environnementale. Cette piste serait celle qui permettrait de penser une convergence entre les éthiques relationnelles de la nature et une version forte de la durabilité au sein d'une approche écocentrique.

Dans le champ des éthiques environnementales, les théories écocentriques se distinguent du biocentrisme par le refus d'adopter l'ontologie individualiste qui accompagne la quête biocentrique des entités vivantes que l'on pourrait isoler et décrire comme des porteurs de valeur intrinsèque. Ces théories sont, en effet, moins préoccupées par la valeur individuelle des êtres vivants que par le sort des communautés socioécologiques auxquelles appartiennent ces derniers (Beau, 2015). De ce point de vue, l'écocentrisme est au fond bien mal nommé, en ce sens qu'il ne renvoie pas tant à un centre vers lequel 
devraient converger les obligations morales qu'au fonctionnement d'ensemble d'un système (Plumwood, 2002: 97-123). Ceci est d'autant plus vrai en ce qui concerne les écocentrismes holistes qui rapportent l'évaluation éthique des comportements des différents membres d'une communauté à leur propension à favoriser la santé de cette communauté (Hess, 2013). Il s'agit donc d'adopter le point de vue de la communauté pour juger de la positivité ou de la négativité des relations diverses qui se nouent entre ses membres humains et non humains. Sous cet angle, il apparaît que l'écocentrisme ne peut se limiter au regard extérieur que porterait un observateur humain sur l'état d'un système naturel, il est aussi une interrogation sur les manières qu'ont les hommes de s'insérer dans les systèmes naturels et d'évoluer au sein de ce qui constitue en réalité des socio-écosystèmes. Autrement dit, l'écocentrisme se développe également comme une réflexion sur les manières qu'ont les êtres humains, parmi d'autres espèces, d'habiter la terre. Ainsi, d'une façon qui pourrait sembler paradoxale, les pensées que l'on a souvent décrites comme étant les plus radicalement non anthropocentristes peuvent néanmoins reconduire en dernière instance vers une réflexion éthique qui inclut les pratiques humaines ${ }^{7}$.

Mais, si ce paradoxe n'est qu'apparent, c'est précisément parce que l'écocentrisme bien conçu nous semble conduire au-delà du dualisme et permettre d'envisager la réintégration des humains dans le champ de la réflexion environnementale sans que celle-ci mène pour autant au recentrement de l'éthique sur les seuls humains. Or, cela est possible parce que ce retour des humains dans l'éthique environnementale est opéré par la description des relations multiples qu'ils nouent avec les animaux, les plantes et l'ensemble des êtres vivants et non vivants, autrement dit parce qu'il s'opère dans le cadre d'une approche relationnelle. C'est bien, en effet, la capacité constitutive donnée aux relations vis-à-vis des êtres moraux qui permet de penser la cohabitation d'un collectif sur un territoire d'une façon qui n'est plus étroitement anthropocentriste. Sur ce point, les travaux respectifs de Baird Callicott (Callicott, 2014) et de Val Plumwood (Plumwood, 2002, 2014) sont décisifs et complémentaires, parce que les premiers parviennent à la formulation la plus convaincante d'un écocentrisme relationnel, né de la rencontre entre l'écologie scientifique et l'éthique, et que les seconds confortent l'éthique relationnelle dans la voie de la durabilité forte.

En ce qui concerne Callicott, la land ethic d'inspiration léopoldienne (Callicott, 2011) qu'il défend peut bien apparaître comme l'expression emblématique d'une éthique environnementale du «tout relié », dont Frédéric Neyrat pointait les dangers. Pour le philosophe, en effet, l'écho du développement de la science écologique doit retentir dans le champ de l'éthique en donnant lieu à la formation d'une ontologie morale relationnelle. Reprenant à son compte la paraphrase de Bruno Latour qui donne son titre à un article co-écrit par deux biologistes et un philosophe (Gilbert et al., 2012), Callicott soutient que nous ne pouvons penser une éthique adéquatement sans commencer par reconnaître que «nous n'avons jamais été des individus", c'est-à-dire des entités indépendantes que l'on pourrait isoler ou délier des autres êtres. Pour le philosophe écocentriste, on ne saurait désencombrer un être moral de ses relations sans le réduire à une conception abstraite de l'homo ethicus, modelée sur celle de l'homo economicus, autrement dit à une version éthique de la figure de l'« idiot rationnel » (Callicott, 2014 : 292). En ce qui concerne l'éthique environnementale, Callicott ne semble donc pas croire en la dimension heuristique de la voie de la séparation des hommes et de la nature, mais 
invite au contraire à partir de la description des relations spéciales qui attachent les êtres entre eux et à un territoire.

31 Cela fait-il de l'écocentrisme de Callicott une forme d'écoconstructivisme dans lequel la nature céderait en définitive le pas à l'humain? Sur le plan de l'éthique environnementale, une telle critique serait de toute évidence injustifiée. Mais, comme nous l'avons vu, la critique des approches relationnelles que nous avons décrite porte au fond principalement sur le plan de l'écologie politique. Or, de ce point de vue, il faut bien reconnaître que l'éthique environnementale de Callicott demande des éclaircissements (Hess, 2017). Le primat donné aux attachements subjectifs dans la pensée environnementale ne fait-il pas courir le risque de perdre de vue les enjeux écologiques majeurs ? Cet écocentrisme relationnel ne conduit-il pas à décrire une réconciliation avec la nature qui n'opérerait que dans le ciel des expériences cosmiques, flottant bien audessus des réalités matérielles de la crise écologique? De ce point de vue, on ne serait sorti du dualisme que pour lui opposer une sorte de monisme idéaliste, assez inoffensif sous l'angle de la critique sociale et écologique.

Mais il s'agit là, à notre sens, d'une interprétation tronquée de l'éthique écocentrique, que permet précisément d'écarter la clarification des rapports de cette dernière avec une approche de la durabilité forte. Que l'on puisse mettre en doute l'existence d'un écart irréductible entre un écocentrisme relationnel et les approches écoconstructivistes décrites par Frédéric Neyrat révèle, en effet, sans aucun doute un manque dans l'élucidation de ce que pourrait être la contribution des éthiques environnementales à une pensée critique relevant du champ de l'écologie politique. Ce manque est, en réalité, celui d'une articulation plus explicite entre deux conceptions de l'interdépendance des hommes et de la nature, qui caractériseraient respectivement les approches éthiques et celles de la durabilité forte. Tandis que les éthiciens de l'environnement mettraient l'accent sur l'interdépendance affective, psychologique, voire spirituelle, des êtres humains et non humains, les tenants de la durabilité forte, et en particulier les représentants du courant de l'économie écologique, se focaliseraient sur la matérialité de cette interdépendance. Schématiquement, les premiers valoriseraient les échanges d'affects au sein d'une communauté mixte d'humains et de non-humains quand les seconds seraient principalement préoccupés par les échanges de matières et d'énergie. Or, cette description, qui prend place dans un cadre dualiste, n'est pas satisfaisante car elle conduit à maintenir séparé ce que l'écologie politique nous semble devoir réunir. C'est précisément ce que défend la philosophe Val Plumwood, notamment dans un chapitre remarquable de son ouvrage Environmental Culture: The Ecological Crisis of Reason, intitulé « Toward a materialist spirituality of place » (Plumwood : 2002, 218-235).

Par cette expression de "spiritualité matérialiste», la philosophe australienne entend s'opposer à une interprétation purement idéaliste de la pensée des attachements des humains à un lieu et veut montrer, au contraire, comment une éthique relationnelle qui accorde une place importante aux émotions rejoint la critique politique de la négation de la dépendance matérielle des humains à l'égard de la nature. Autrement dit, elle permet de penser la convergence entre une éthique environnementale relationnelle et une conception de la durabilité forte. Pour Plumwood, un lieu se définit par l'histoire commune des habitants humains et non humains d'un espace, et est composé par l'ensemble des relations tissées entre ces êtres. Mais ces attachements ne renvoient pas simplement à l'abstraction d'une sorte de communion spirituelle entre les êtres. Dès le début des années 1990, Val Plumwood clarifiait ce point: 
«Or, pareil attachement n'est pas fondé sur une vague théorie cosmologique, abstraite et purement cérébrale, mais sur la formation d'une identité, sociale et personnelle, en relation avec certains endroits particuliers d'une région, qui crée des liens souvent aussi spécifiques et aussi puissants que ceux de la parenté, et leur sont analogues en ceci qu'ils suscitent des responsabilités locales très spécifiques envers les lieux dont il convient de prendre soin » (Plumwood, 2014).

C'est cet entrelacs d'attachements qui rend par définition un lieu insubstituable. Il est le produit d'une histoire faite d'expériences vécues et partagées sur un même espace. Cette définition du lieu prend bien sûr à contre-pied l'hypothèse de substituabilité qui a soustendu la libéralisation du marché de la terre (Polanyi, 1983(1944)). Elle conduit ainsi de façon très claire à se positionner contre un projet écoconstructiviste ancré dans une économie néolibérale, «si habile à couper les objets et les sujets de leurs attachements sociaux et écologiques pour les reformater indéfiniment en marchandises circulant dans de nouveaux réseaux» (Bonneuil, 2015).

Mais, la théorie des attachements de Plumwood ne consiste pas simplement à opposer le caractère insubstituable des expériences vécues de la nature faites par les hommes à la fongibilité supposée des êtres naturels permettant leur marchandisation. En premier lieu, contre l'anthropocentrisme que ferait resurgir une telle description, la philosophe souligne à plusieurs reprises la part active que prennent les habitants non humains d'un espace dans la construction d'un lieu (Plumwood, 2002: 231). Ensuite, cette opposition resterait par trop indifférenciée pour incarner une pensée critique. La description des interrelations composant une communauté d'habitants humains et non humains sur un territoire, qui nous semble pouvoir caractériser l'écocentrisme relationnel, ne doit pas occulter le fait que des disparités profondes existent quant à la capacité des différents membres de cette communauté à faire valoir leurs différents attachements. Sur ce point, Plumwood précise :

«Les approches hyper-individualistes, qui suggèrent que le capitalisme libéral moderne peut promouvoir l'attachement à un lieu, négligent la façon dont les relations à un lieu sont différenciées et structurées à travers plusieurs relations de genre et de race, et sont affectées aussi par le statut de classe et le statut colonial ${ }^{8}$ » (Plumwood, 2002 : 233-234).

De ce point de vue, la philosophe déploie la dimension politique de son éthique relationnelle. Si la réflexion sur la durabilité forte consiste à mener une interrogation sur ce qu'un collectif veut instituer comme insubstituable, cette institution est un processus politique au cours duquel les différents membres d'un collectif défendent leurs attachements sociaux et écologiques. Or, cette recherche d'un accord sur l'insubstituable se tient dans un contexte marqué par de graves inégalités environnementales et sociales (Larrère, 2017). Elle est par conséquent nécessairement traversée de conflits et ne renvoie aucunement à la conception irénique d'une coopération harmonieuse des différents habitants sur un même espace. Cette éthique relationnelle ouvre plutôt une voie démocratique permettant de mettre les attachements d'un collectif au service de la critique d'une société fondée sur la croissance qui nie l'existence de ses limites sociales (Hirsch, 2016) et écologiques. Dans ce sens, Val Plumwwood écrit :

«La quête d'une société sensible à la nature et au lieu, comme la quête pour une meilleure qualité de vie professionnelle et pour une démocratie réellement communicationnelle, dévoile un projet qui est radical en ce sens que son accomplissement remettrait en cause fondamentalement et très profondément l'ordre existant à plusieurs niveaux ${ }^{9} »($ Plumwood, $2002: 235)$. 


\section{Conclusion}

De la rencontre entre les éthiques environnementales et la réflexion sur la durabilité peut ainsi naître ce que l'on pourrait appeler une «théorie écocentrique de la durabilité forte » (Lejeune et Villalba, 2015). Tout en s'appuyant sur une éthique environnementale relationnelle, cette approche doit tenir compte de la critique importante développée par des défenseurs du dualisme. Elle doit donc se défier des conséquences politiques d'une pensée des interrelations entre les humains et les non-humains qui basculerait dans un hybridisme dont les affinités sont fortes avec la néolibéralisation du monde. Il lui faut penser une intégration des activités humaines dans des systèmes écologiques, sans que ces derniers disparaissent derrière le manteau épais des constructions sociales. L'écocentrisme permet d'envisager un enchâssement de niveaux d'organisation collective, comme autant de systèmes de relations que des collectifs peuvent vouloir rendre insubstituables en droit. Certaines évolutions récentes de systèmes juridiques témoignent de cette possibilité. C'est ainsi, en effet, que la reconnaissance de la personnalité juridique attribuée au fleuve Whanganui par le parlement néo-zélandais en mars 2017, qui le qualifie par-là même d'« être vivant unique » - insubstituable donc peut être considérée comme la concrétisation juridique de la lutte politique menée par les Maoris afin de défendre l'ensemble des relations sociales et écologiques qui les associent au fleuve et à ses habitants non humains. Nous voulons y voir les prémices de l'expression politique d'un écocentrisme relationnel.

\section{BIBLIOGRAPHIE}

Afeissa H.-S. éd., 2007, Éthique de l'environnement : nature, valeur, respect, Paris, Vrin.

Arpin I., 2016, «Les sources de l'éthique : diversité des pratiques et recherches d'unité », colloque Quelles éthiques pour les relations humains-biodiversité ?, 9 et 10 décembre 2016, université Paris 1 Panthéon-Sorbonne.

Asafu-Adjaye J., Blomqvist L., Brand S., Brook B. W., DeFries R., Ellis E., Nordhaus T., Foreman Ch., Keith D., Lewis M. Lynas M., Roger Pielke J., Pritzker R., Ronald P., Joyashree R., Sagoff M., Shellenberger M., Stone R., Teague P., 2015, « An Ecomodernist Manifesto », http:// www.ecomodernism.org/manifesto, consulté le : 21 octobre 2016.

Barde P., 1992, Économie et politique de l'environnement, $2^{\mathrm{e}}$ éd., Paris, PUF.

Beau R., 2015, « Écocentrisme », in Bourg D., Papaux A. (dir.), Dictionnaire de la pensée écologique, Paris, PUF, p. 307-309.

Beau R., 2017, Éthique de la nature ordinaire : recherches philosophiques dans les champs, les friches et les jardins, Paris, Publications de la Sorbonne.

Beckerman W., 1995, Small Is Stupid : Blowing the Whistle on the Greens, London, Duckworth. 
Beckerman W., 1994, «"Sustainable development” : is it a useful concept ? ", Environmental values, vol. 3, n 3, p. 191-209.

Bonneuil C., 2015, « Chapitre 3. Comment ne pas voir les limites de la planète », in Sinaï A. (dir.), Économie de l'après-croissance, Paris, Presses de Sciences Po, p. 73-96.

Brundtland G. H., 1987, « Notre avenir à tous, rapport de la commission mondiale sur l'Environnement et le Développement », Les Éditions du Fleuve, Paris (traduction française de Our Common Future.

Callicott J. B., 2016, « 22. Ecological sustainability », The Routledge Handbook of Philosophy of Biodiversity, New York, Routledge, p. 311-325.

Callicott J. B., 1999, Beyond the Land Ethic : More Essays in Environmental Philosophy, New York, University of New York Press.

Callicott J. B., 1989, In Defense of Land Ethic : Essays in Environmental Philosophy, New York, University of New York Press.

Callicott J. B., Mumford K., 1997, « Ecological Sustainability as a Conservation Concept », Conservation Biology, vol. 11, n 1, p. 32-40.

Callicott J. B., Nelson M. P., 1998, The Great New Wilderness Debate, Athens, University of Georgia Press.

Callicott J. B., 2011, Éthique de la terre, Paris, Wildproject.

Callicott J. B., 1998, «The Wilderness Idea Revisited. The Sustainable Development Alternative », in Callicott J. B., Nelson M. P. (dir.), The Great New Wilderness Debate, Athens, University of Georgia Press, p. 337-366.

Callicott J. B., 2014, Thinking Like a Planet : The Land Ethic and the Earth Ethic, Oxford, Oxford University Press.

Callicott J. B., Nelson M. P, 2008, The wilderness debate rages on : continuing the great new wilderness debate, Athens, University of Georgia Press.

Cobb J., Daly H., 1989, For the common good, redirecting the economy toward community, the environment and a sustainable future, Boston, Beacon Press.

Costanza R., 1991, Ecological Economics : The Science and Management of Sustainability, New York, Columbia University Press.

Cronon W., 1996, « The trouble with wilderness : or, getting back to the wrong nature ", Environmental History, vol. 1, $\mathrm{n}^{\circ}$ 1, p. 7-28.

Daly Herman E., 1995, « On Wilfred Beckerman's Critique of Sustainable Development », Environmental Values, vol. 4, n 1, p. 49-55.

Ekins P., 2003, « Identifying critical natural capital : Conclusions about critical natural capital », Ecological Economics, vol. 44, n² 2, p. 277-292.

Gilbert Scott F., Sapp Jan, Tauber A. I., 2012, « A symbiotic view of life : we have never been individuals ", The Quarterly review of biology, vol. 87, $\mathrm{n}^{\circ} 4$, p. 325-341.

Hess G., 2013, Éthiques de la nature, Paris, PUF.

Hess G., 2017, « Réconcilier l'éthique environnementale et l'écologie politique : une analyse métaéthique »,. La pensée écologique, https://lapenseeecologique.com/reconcilier-lethiqueenvironnementale-et-lecologie-politique-une-analyse-meta-ethique, consulté le 7 mars 2018. 
Hirsch F., 2016, Les limites sociales de la croissance, Paris, Les Petits Matins.

Holland A., 2002, « Or, why strong sustainability is weak and absurdly strong sustainability is not absurd », in Foster J. (dir.), Valuing Nature ? : Economics, Ethics and Environment, New York, Routledge, p. 119.

Jamieson D., 1998, « Sustainability and beyond », Ecological Economics, vol. 24, n 2, p. 183-192.

Katz E. et Light A. (dir.), 1996, Environmental Pragmatism, $1^{\text {re }}$ éd., New York, Routledge.

Larrère C., 2002, «Avons-nous besoin d'une éthique environnementale ? », Cosmopolitiques. La nature n'est plus ce qu'elle était, vol. , n 1, p. 69-85.

Larrère C., 2017, Les inégalités environnementales, Paris, PUF.

Larrère C., 1997, Les philosophies de l'environnement, Paris, PUF.

Lejeune C., Villalba B., 2015, « Délibérer à partir de l'environnement. Une perspective théorique écocentrée de durabilité forte ", Environnement : la concertation apprivoisée, contestée, dépassée, Mermet L. et Salles D. (dir.), Louvain-La-Neuve, De Boeck Superieur, p. 257-270.

Leopold A., 2000, Almanach d'un comté des sables : suivi de quelques croquis, Paris, Flammarion.

Light A., Rolston H. (dir.), 2002, Environmental Ethics : An Anthology, $1^{\text {re }}$ éd., Hoboken, WileyBlackwell.

Maris V., 2015, «Back to the Holocene : a conceptual, and possibly practical, return to a nature not intended for humans », in Hamilton C., Gemenne F., Bonneuil C. (dir.), The Anthropocene and the Global Environmental Crisis : Rethinking Modernity in a New Epoch, New York, Routledge, p. 123-133.

Maris V., 2014, Nature à vendre : Les limites des services écosystémiques, Versailles, Quæ.

Martinez-Alier J., 1990, Ecological Economics : Energy, Environment and Society, Oxford, WileyBlackwell.

Meadows D. H., Meadows D. L., Randers J. et Behrens W., 1972, Halte à la croissance ? Rapport sur les limites de la croissance, Paris, Fayard.

Merchant C., 2004, Reinventing Eden: The fate of nature in Western culture, London, Routledge.

Naess A., 1989, Ecology, Community, and Lifestyle: Outline of an ecosophy, Cambridge, Cambridge University.

Nash R., 1967, Wilderness and the American Mind, New Haven, Yale University Press.

Neyrat F., 2016, La part inconstructible de la terre. Critique du géo-constructivisme, Paris, Seuil.

Neyrat F., 2017, « Nature, anaturalisme, et géoconstructivisme - La pensée écologique », Dictionnaire de la pensée écologique, http://lapenseeecologique.com/entree-nature-anaturalismeet-geo-constructivisme, consulté le 9 novembre 2017.

Norton B. G., 1984, « Environmental Ethics and Weak Anthropocentrism », Environmental Ethics, vol. $6, \mathrm{n}^{\circ}$ 2, p. 131-148.

Norton B. G., 2003, Searching for Sustainability: Interdisciplinary Essays in the Philosophy of Conservation Biology, New York, Cambridge University Press.

Norton B. G., 2005, Sustainability: A Philosophy of Adaptive Ecosystem Management, Chicago, University of Chicago Press. 
Norton B. G, 2008, « Why I am Not a Nonanthropocentrist », Environmental Ethics, vol. 17, n 4 , p. 341-358.

Noss R. F., 1991, «Sustainability and Wilderness », Conservation Biology, vol. 5, n 1, p. 120-122.

Plumwood V., 2002, Environmental Culture : The Ecological Crisis of Reason, London, Routledge.

Plumwood V., 2014, « La nature, le moi et le genre », Écologie \& politique, vol. $\mathrm{N}^{\circ} 48, \mathrm{n}^{\circ} 1$,

p. $143-175$.

Plumwood V., 1998, « Wilderness Skepticism and Wilderness Dualism », The Great New Wilderness Debate, Callicott J. B. et Nelson M.P. (dir.), Athens, University of Georgia Press, p. 652-690.

Polanyi K., 1983 (1944), La Grande transformation : aux origines politiques et économiques de notre temps , Paris, Gallimard.

Rolston H., 1989, Philosophy Gone Wild, Buffalo, New York, Prometheus Books.

Rolston III H., 2002a, « Justifying Sustainable Development : A continuing ethical search »,. Global Dialogue, vol. 4, $\mathrm{n}^{\circ}$ 1, p. 103.

Rolston III H., 2002b, « Naturalizing Callicott », in Ouderkirk W. et Hill J. (dir.), Land, Value, Community: Callicott and Environmental Philosophy, p. 107-122.

Rolston III H., 2008, « The Wilderness Idea Reaffirmed », in Nelson M.P, Callicott J. B. (dir.), The Wilderness Debate Rages On : Continuing the Great New Wilderness Debate, Athens, University of Georgia press, p. 367-386.

Shellenberger M., Nordhaus T., 2011, Love Your Monsters : Postenvironmentalism and the Anthropocene, Oakland, Breakthrough Institute.

Solow R. M., 1993, "Sustainability : An economist's perspective ", Economics of the environment : selected readings, New York, Norton, p. 179-187.

Solow R. M., 1974, "The Economics of Resources or the Resources of Economics », The American Economic Review, vol. 64, n 2, p. 1-14.

Taylor P. W., 1986, Respect for Nature : A Theory of Environmental Ethics, Princeton, Princeton University Press.

Vivien F.-D., 2004, « Un panorama des propositions économiques en matière de soutenabilité », Vertigo - la revue électronique en sciences de l'environnement, vol. $5, \mathrm{n}^{\circ} 2, \mathrm{http}: / /$

journals.openedition.org/vertigo/3620, consulté le 23 février 2018.

Vucetich J. A., Nelson M. P., 2010, « Sustainability : Virtuous or Vulgar ? », BioScience, vol. 60, n 7, p. 539-544.

\section{NOTES}

1. Sur la distinction entre une économie de l'environnement et une économique pour l'environnement, voir Vivien (2004).

2. Pour une présentation détaillée de ce courant, voir Larrère (1997); Afeissa (2007); Hess (2013).

3. Norton, lui-même, se présente comme l'un des éthiciens de l'environnement les plus à même d'entendre les arguments des économistes, (Norton, 2003 : 165-167).

4. Ce que Bryan Norton décrit comme une erreur grossière, "a fool's errand from the start ", (Norton, $2003:$ 431).

5. Nous renvoyons là au titre de Frédéric Neyrat (2016) sur lequel nous allons revenir. 
6. Voir l'expression de sharp ecology développée par Neyrat (2016).

7. Ce «paradoxe» a été souligné avec justesse par Gérald Hess dans l'introduction de son chapitre consacré aux écocentrismes holistes (Hess, $2013: 304$ ).

8. "Over-individualised accounts which suggest modern liberal capitalism can promote place attachment, overlook the way relationships to place are differentiated and structured through various gendered and racialised relationships, and are affected also by class and colonial status. » [Notre traduction]

9. «The quest for a nature and place-sensitive society, like the quest for a better quality working life and a genuinely communicative democracy, unveils a project that is radical in the sense that its fulfilment as the normal case would challenge the existing order very deeply and fundamentally at many levels. " [Notre traduction].

\section{RÉSUMÉS}

Le débat sur les durabilités fortes et faibles a souvent été perçu par les représentants du courant des éthiques environnementales comme un débat d'économistes auquel ils ne souhaitaient pas prendre part. Critiquant l'anthropocentrisme sous-jacent à ces réflexions articulées autour de la notion de "capital naturel », les éthiciens de l'environnement ont défendu l'idée que les enjeux de la préservation de la nature conduisaient bien au-delà de la réflexion sur la durabilité des sociétés humaines. Largement partagée au sein du courant, cette critique n'empêcha pas néanmoins certains auteurs de proposer une approche philosophique de la durabilité écologique. C'est cet engagement dans la réflexion sur la durabilité par des éthiques environnementales relationnelles que nous examinons dans cet article, afin de dessiner les contours d'une approche écocentrique de la durabilité forte.

The debate between strong and weak sustainability was often described by the environmental ethicists as a debate between economists to which they didn't want to participate in. When criticizing the anthropocentric notion of «natural capital », environmental philosophers have defended that the goals of nature preservation needed to go far beyond the question of the sustainability of human societies. However, this criticism didn't prevent some environmental ethicists from proposing a philosophical approach of ecological sustainability. We particularly analyse here some relational environmental ethics through the lenses of the debate on sustainability in order to define an ecocentric approach of strong sustainability.

\section{INDEX}

Keywords : strong sustainability, environmental ethics, ecocentrism, ordinary nature, relational ethics, non-substituability, environmental philosophy

Mots-clés : durabilité forte, éthique environnementale, écocentrisme, nature ordinaire, éthique relationnelle, insusbstituabilité, philosophie de l'environnement 


\section{AUTEUR}

RÉMI BEAU

Rémi Beau est docteur en philosophie de l'université Paris 1 Panthéon-Sorbonne. Spécialiste des éthiques et de la philosophie environnementales, il s'intéresse tout particulièrement aux relations que nous entretenons avec la nature proche ou ordinaire, nature avec laquelle nous vivons et interagissons au quotidien. 